\title{
Digital Heterodyne Holography Reveals the Non-Quasi-Static Scattering Behaviour of Transversally Coupled Nanodisk Pairs
}

\author{
Sarah Y. Suck, ${ }^{1,2}$ Sébastien Bidault, ${ }^{1}$ Nicolas Bonod, ${ }^{3}$ Stéphane Collin, ${ }^{4}$ \\ Nathalie Bardou, ${ }^{4}$ Yannick De Wilde, ${ }^{1}$ and Gilles Tessier ${ }^{1}$ \\ ${ }^{1}$ Institut Langevin, ESPCI ParisTech, CNRS UMR 7587, 10 rue Vauquelin, 75231 Paris Cedex 05, France \\ ${ }^{2}$ Fondation Pierre-Gilles de Gennes pour la Recherche, 29 rue d'Ulm, 75005 Paris, France \\ ${ }^{3}$ Institut Fresnel, CNRS UMR 7249, Aix-Marseille Université, Ecole Centrale Marseille, Domaine Universitaire de Saint Jérôme, \\ 13397 Marseille, France \\ ${ }^{4}$ Laboratoire de Photonique et de Nanostructures (LPN-CNRS UPR 20), route de Nozay, 91460 Marcoussis, France
}

Correspondence should be addressed to Gilles Tessier, gilles.tessier@espci.fr

Received 27 October 2011; Accepted 9 December 2011

Academic Editor: Alexandre Bouhelier

Copyright (c) 2012 Sarah Y. Suck et al. This is an open access article distributed under the Creative Commons Attribution License, which permits unrestricted use, distribution, and reproduction in any medium, provided the original work is properly cited.

\begin{abstract}
We reconstruct the full three-dimensional scattering pattern of longitudinal and transverse modes in pairs of coupled gold nanodisks using digital heterodyne holography. Near-field simulations prove that, in our experimental conditions, the induced dipoles in the longitudinal mode are in phase while they are nearly in opposite phase for the transverse mode. The scattering efficiency of the two modes is of the same order of magnitude, which goes against the common belief that antisymmetric transverse modes are "dark." The analysis of the reconstructed hologram in the Fourier plane allows us to estimate the angular scattering pattern for both excited modes. In particular, the antisymmetric transverse mode scatters light mostly into one halfplane, demonstrating that the quasi-static approximation breaks down in nanodisk pairs even for an interparticle distance lower than $\lambda / 4$.
\end{abstract}

\section{Introduction}

The coupling of two nanoparticles plays an important role in the context of plasmonics because of the increased nearfield enhancement and confinement that occurs in the gap between particles. Pairs of coupled nanoparticles represent the simplest and most fundamental structure for studying the plasmon coupling phenomena which give rise to numerous applications like surface-enhanced Raman spectroscopy (SERS) $[1,2]$, nanosensing [3-5], and optical trapping $[6,7]$.

The resonances and optical properties of a two-particle antenna are often described in terms of the hybridization model which states that the coupling of individual particle dipole modes induces an energy splitting into bonding and antibonding modes [8]. This quasi-static model entails that the red-shifted bonding longitudinal mode and the blue-shifted antibonding transverse mode are the only ones that couple efficiently to far-field radiation. These modes are generally called "bright" modes while the antibonding longitudinal and bonding transverse modes are described as nonscattering "dark" modes and are, thus, not observed in the far field.

With the increasing development in nanofabrication technology during the last decade, control over the design of two-particle antennas has been steadily improving. Since then, the coupling phenomena involved in the optical properties of a two-disk nanostructure have been studied extensively using experimental and numerical approaches [9-12]. In all these cases, the phase of the excitation is homogeneous on the particle dimers; thus, the spectral responses are consistent with the quasi-static hybridization model.

However, the study of chains of nanoparticles that span distances much greater than the vacuum wavelength has consistently shown that quasi-static approximations break down rapidly in nanofabricated samples $[13,14]$. The retardation effects, which cannot be neglected at these length scales, have promising applications in the design of directional antennas 
(in particular in Yagi-Uda geometries) for the control of single quantum emitters [15-19]. Recently, the directivity of Yagi-Uda nanoantennas has been studied by eigenmode imaging of amplitude and phase using apertureless scanning near-field optical microscopy [20].

In order to reveal the near-field phase effects that drive the performance of optical antennas, we investigate experimentally the optical properties of one of the simplest plasmon coupled systems: a gold nanodisk pair with an interparticle distance much smaller than the vacuum wavelength. Theoretical and experimental reports have shown that at large interparticle distances, retardation effects induce a periodic modulation of the scattering cross-section for both longitudinally and transversely coupled particles [21]. Furthermore, experimental results obtained recently on particle pairs using an excitation in total internal reflection geometry showed that two efficiently scattering transverse modes can be observed [22] which is inconsistent with the hybridization model. In 2011, Rolly et al. studied analytically the optical properties of silver nanogap antennas [23] and have shown that, even at short interparticle distances, phase effects in the induced dipoles have to be taken into account when illuminating the dimer in a direction parallel to its axis. This is not only due to retardation but also to an interparticle scattering term that arises from distance-dependent dipole coupling. In particular, they showed that the maximum scattering efficiency for nearly touching spheres occurs when exciting the antisymmetric (bonding) transverse mode. This mode which is "dark" in the quasi-static approximation is rendered "bright" by the interparticle scattering term. As demonstrated in bimetallic particle pairs, near-field phase effects strongly modify the angular pattern of the scattered light [24]. Three-dimensional (3D) analysis of the scattering pattern of gold dimers should directly discriminate cases where the hybridization model holds.

Digital heterodyne holography, a sensitive technique that relies on interferometry, has the ability to measure the optical amplitude and phase of light scattered by nanoobjects $[25,26]$. Therefore, one hologram which can be recorded in less than 1 second contains the whole $3 \mathrm{D}$ information of the recorded scattered light. Furthermore, holography also allows the access to the angular scattering pattern of a nanostructure since the angular spectrum of the hologram can be obtained by computing the scattered field in the Fourier plane. To correlate measured optical data to the relative phases of the induced dipoles in the particle pair, we performed finite element method (FEM) simulations (based on the commercial software package "Comsol Multiphysics"). This allows us to compute the near-field intensity and phase maps of gold nanodisk pairs in the presence of a glass-air interface.

\section{Simulated Near-Field Maps}

We have developed a three-dimensional simulation model taking into account the presence of the glass-air interface, which is in many cases neglected or simplified in simulations due to its additional complexity when regarding scattering. In our model, we chose to describe the incident wave field in the whole volume, using the analytical expressions of the Fresnel equations describing the diffraction at an interface. The incident light comes from the glass substrate under an incident angle of $45^{\circ}$, which corresponds to the total internal reflection (TIR) configuration used in the experiment.

We were interested in examining the scattered near field of a pair of gold nanodisks in the presence of a glass-air interface under two different excitations using spolarized light: (a) incident $k$-vector perpendicular to the principal axis (i.e., along the $x$-axis) of the nanostructure with an electric field $E_{\mathrm{x}}$ along its axis and (b) incident $k$-vector parallel to its principal axis where the field $E_{\mathrm{y}}$ is perpendicular to the structure. Both configurations are schematically depicted on the left of Figure 1. In case (a), the two disks interact by means of near-field coupling, and the in-phase longitudinal mode is excited, giving rise to a strong field enhancement in the gap. In case (b), in contrast, the near-field coupling of the two particles is weak, as the regions of high near fields are away from the gap between the disks. A transverse mode is excited.

Figure 1 plots the near-field intensity maps in the $x-y$ plane, $\left|E_{x}^{s c}\right|^{2}+\left|E_{y}^{s c}\right|^{2}$ (left images) and the phase maps of the corresponding dominant near-field component, that is, $\Phi\left(E_{x}^{\mathrm{sc}}\right)$ for case (a) and $\Phi\left(E_{y}^{\mathrm{sc}}\right)$ for case (b) (right images). The near-field is calculated $10 \mathrm{~nm}$ above the disks surface to avoid field discontinuities on the gold interfaces and to better correspond to scanning near-field measurements.

The near-field intensity image corresponding to case (a) confirms the excitation of a longitudinal mode. There is a strong field confinement in the gap, which corresponds to an intensity near-field enhancement of 140 . The phase image clearly shows that both disks are in phase. In case (b), we observe no field confinement in the gap, which confirms the excitation of a transverse mode. The nearfield intensity image reveals a slight asymmetry close to the surface of the disks' sides: the near field close to the right disk is higher than that close to the left disk. This feature is due to the incoming field impinging on the right particle from the positive $x$ direction. A possible mode is sketched in the left-handed scheme in Figure 1(b). Regarding the corresponding phase image, both disks are nearly at opposite phase $(\Delta \Phi=1.28 \pi)$ which proves that the disk pair exhibits an antisymmetric transverse mode. Using an illumination in total internal reflection, it will therefore be possible to excite an in-phase longitudinal mode and an opposite-phase transverse mode in the pair of coupled disks and compare their scattering behaviours using digital heterodyne holography. Isotropic gold particles should allow the investigation of the opposite-phase transverse mode as shown in the literature [22]. However, for p-polarized incident light, the strong component of the evanescent field normal to the sample plane forbids the excitation of the symmetric transverse and antisymmetric longitudinal modes in gold disk pairs.

\section{Experimental Setup}

The experimental holographic setup is based on a MachZehnder interferometer. It is schematically depicted in 

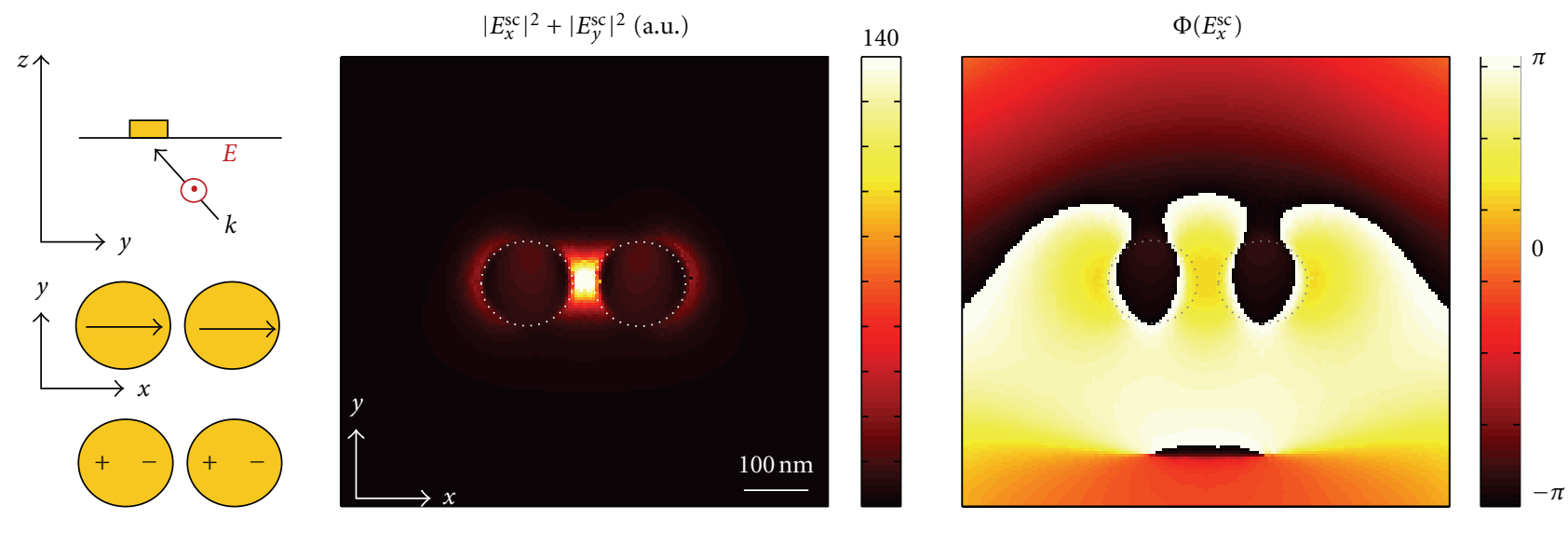

(a)
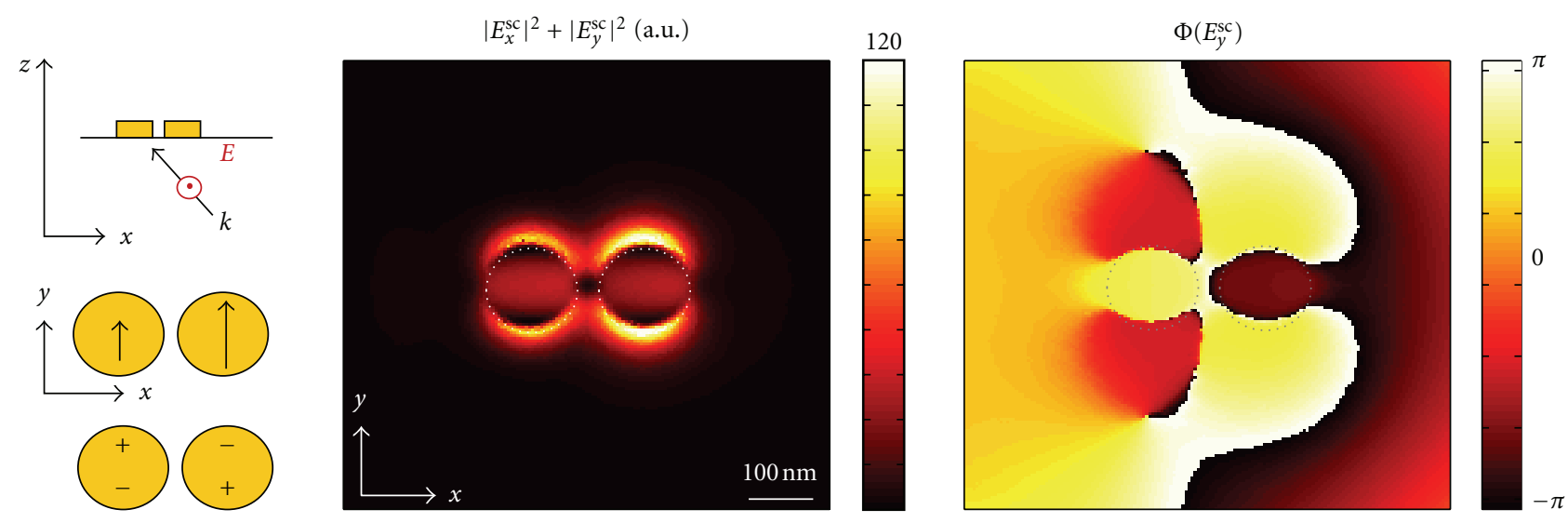

(b)

FIgURE 1: Simulation of the near-field of two coupled disks $(d=150 \mathrm{~nm}$, gap $=40 \mathrm{~nm})$ for 2 different illumination configurations using s-polarized light: (a) wave vector $\perp$ to dimer axis, (b) wave vector $\|$ to dimer axis. Note that only the in-plane components are plotted, that is, the $x$ - and $y$-components. Left: near-field intensity $\left|E^{\text {sc }}\right|^{2}=\left|E_{x}^{\text {sc }}\right|^{2}+\left|E_{y}^{\text {sc }}\right|^{2}$ in the $x$ - $y$ plane plotted 10 nm above the surface of the disks. Right: corresponding phase images. The position of the disks is indicated by dotted circles.

Figure 2. A single mode laser diode $(\lambda=785 \mathrm{~nm}$, Power $=80 \mathrm{~mW}$, Sanyo DL7140-201S) beam is separated with a polarizing beam splitter (PBS) in reference $\left(E_{R}\right)$ and object arms $\left(E_{O}\right)$ which are frequency shifted by two acoustooptical modulators (AOM1, AOM2) at frequencies $f_{\mathrm{AOM} 1}$ and $f_{\mathrm{AOM} 2}$ creating an accurate phase shift. Half wave plates $(\lambda / 2)$ and neutral density filters (ND1, ND2) allow the control of the optical power in each arm. Two lens systems are introduced in the setup $(L 1, L 2)$. An expanded reference beam fully illuminates the CCD camera (Roper Cascade $512 \mathrm{~F}$ EMCCD, frame transfer $512 \times 512,16 \times 16 \mu \mathrm{m}$ pixel matrix, triggered at a frame rate $f_{\mathrm{CCD}}=16 \mathrm{~Hz}$, integration time $t_{\text {int }}=50 \mathrm{~ms}$ ). The glass substrate of the sample is coupled to a prism using an index matching liquid and illuminated in TIR configuration to achieve dark field illumination. The scattered field is collected by a microscope objective (MO, 100x magnification, $\mathrm{NA}=0.95$ in air) and reaches the CCD camera where it interferes with the reference beam in an off-axis manner. The resulting interference pattern is modulated at a beat frequency $\Delta f=$ $f_{\mathrm{AOM} 1}-f_{\mathrm{AOM} 2}$, and the hologram is recorded by the camera at a rate $f_{\mathrm{CCD}}=4 \Delta f$. The holograms presented here are obtained with an acquisition time of 2 seconds. The reconstruction of the hologram is achieved numerically after $k$ space filtering using a method inspired by Cuche et al. [27]. The off-axis configuration, combined with heterodyne modulation, allows spatial and temporal filtering, yielding excellent signal-to-noise ratios and sensitivities $[25,28]$.

The gold nanostructures under study have been fabricated by electron beam lithography onto a glass substrate. The two gold nanodisks have a thickness of $30 \mathrm{~nm}$ with an additional $2 \mathrm{~nm}$ thick chromium (Cr) wetting layer, a diameter $d$ of $150 \mathrm{~nm}$ and are separated by an interdisk spacing of $40 \mathrm{~nm}$. Since we use $785 \mathrm{~nm}$ excitation, the nanodisk pairs feature strongly subwavelength dimensions with a $\lambda / 4$ center-to-center spacing.

We investigated the far-field spectral response of the nanostructures using a white light source in dark field illumination and an imaging spectrometer, as described in Suck et al. [26]. Figure 3 plots the normalized scattering intensity spectrum of the longitudinal mode of a pair of coupled disks in comparison with the spectrum of a single 


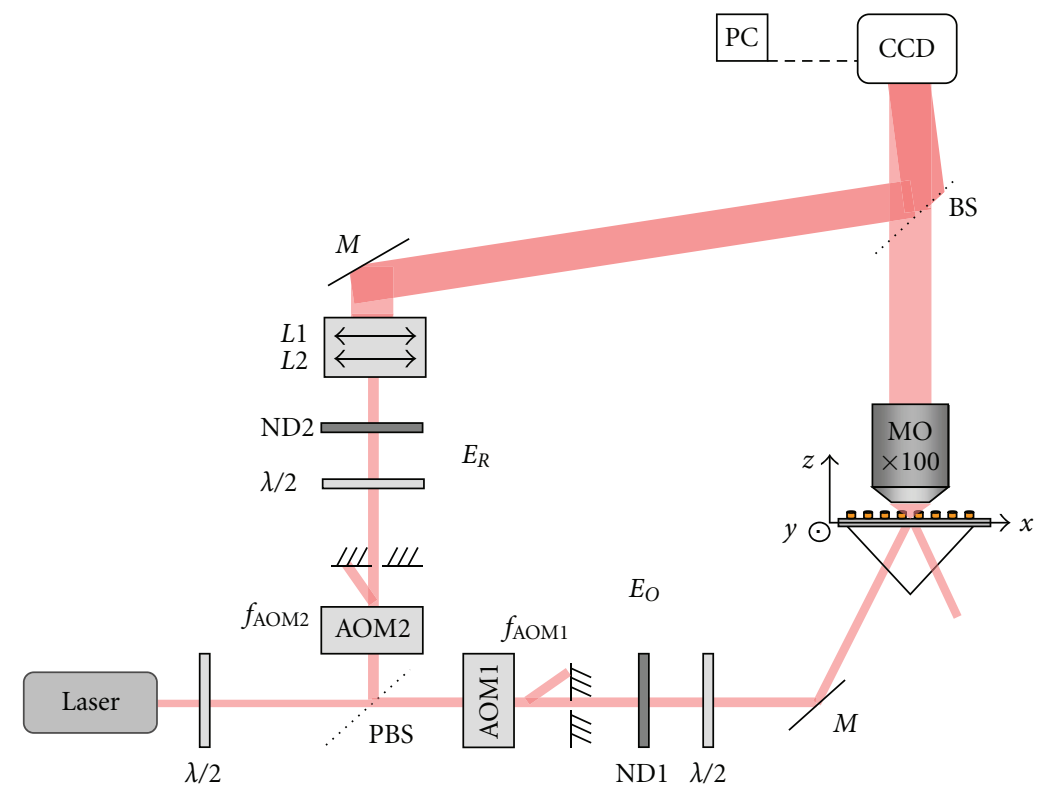

FIGURE 2: Experimental setup based on heterodyne off-axis holography. See text for details and abbreviations.

disk. The longitudinal mode is excited using an incoming polarization parallel to the dimer axis. The resonance wavelength at $760 \mathrm{~nm}$ is close to the excitation wavelength used in the holographic setup. The symmetric transverse mode resonance is expected to be close to the single disk resonance $[9,10]$.

\section{Far-Field Scattering Maps of Two Coupled Disks}

In order to excite two different modes in the coupled disks system, we used the two different illumination configurations as described above. Figure 4 presents cross sections along the three main reconstruction planes $(x-y, y-z$, and $x-z$ planes $)$ of far-field scattering images obtained by digital heterodyne holography on two coupled disks. The position of the nanostructure with respect to two different planes of incidence $(y-z$ for case (a) and $x-z$ for case (b)) is schematically shown on top of each image. Note that the planes containing the $z$ axis are plotted using a logarithmic scale. When analysing the holographic images, cross sections along the $z$-axis should be interpreted with care for negative $z$-values: the holographic signal only collects forward scattered light, and the intensity shown in the glass substrate $(z<0)$ only corresponds to backpropagation of this light without a direct physical meaning.

Analysing the scattering characteristics in the three reconstruction planes, it is seen that in both cases the scattering maps in the $x-y$ plane show a bright dot with a size of around $500 \mathrm{~nm}$. Cross-sections containing the $z$-axis indicate that most of the scattered light is distributed along the $z$-axis in the half space of air. The measured scattered intensities in both excitation geometries are of the same order of magnitude, indicating that both induced modes couple to the far-field with comparable efficiencies. The in-phase

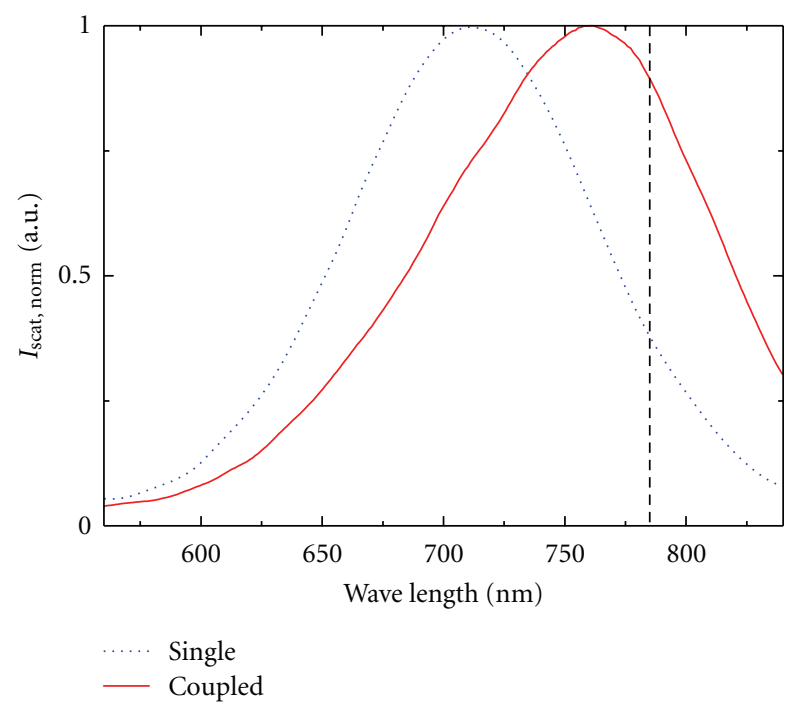

FIGURE 3: Normalized scattered intensity $I_{\text {scat,norm }}$ spectra of a single disk, $d=150 \mathrm{~nm}$ (dotted spectrum), and a pair of coupled disks, $d=150 \mathrm{~nm}$, gap $=40 \mathrm{~nm}$ (continuous spectrum). The straight dashed line denotes the position of the exciting wavelength, $\lambda_{\mathrm{exc}}=$ $785 \mathrm{~nm}$.

longitudinal mode has only a scattering efficiency between 2 and 3 times larger than the antisymmetric transverse mode, even though the excitation wavelength is close to the resonance of the longitudinal mode. According to analytical calculations, it should be possible to render the transverse mode brighter than the longitudinal mode by reducing the interparticle spacing and tuning the excitation wavelength [23].

Furthermore, we observe that the far-field scattering pattern of the transverse mode differs slightly from the 

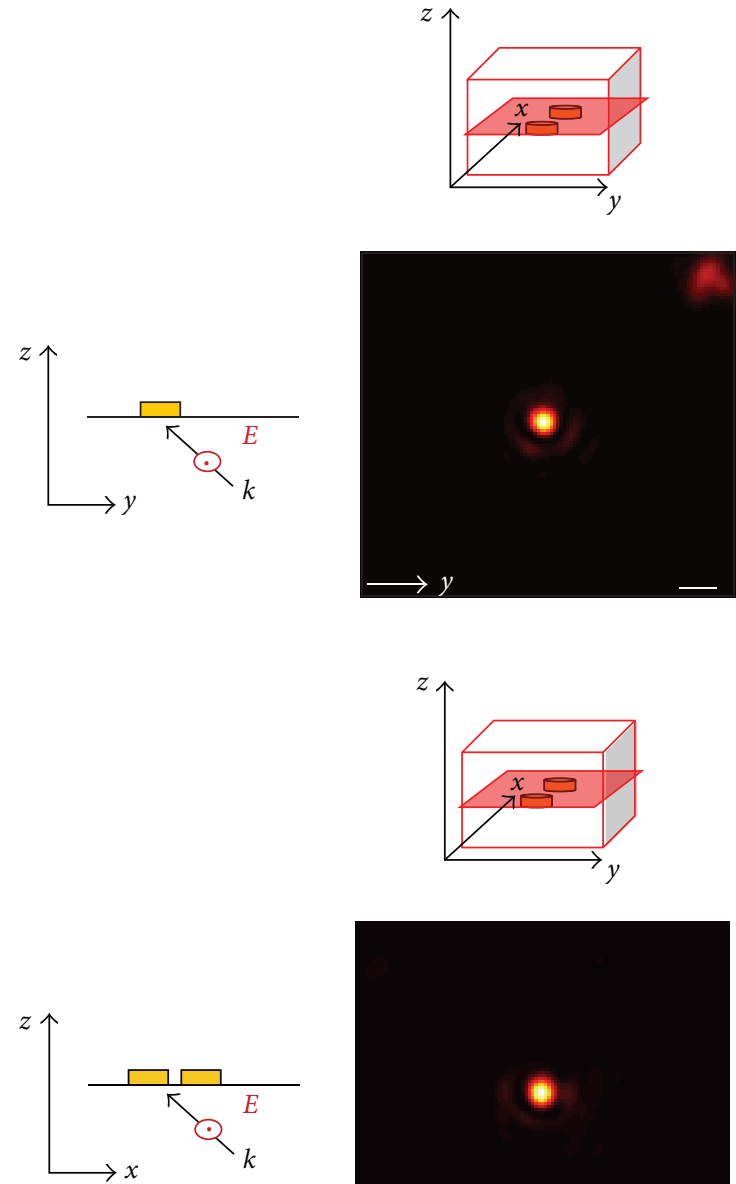
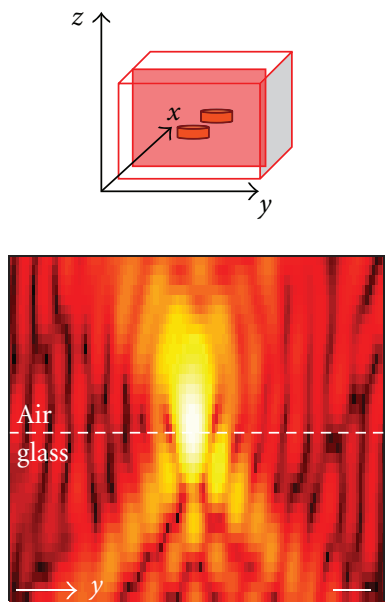

(a)
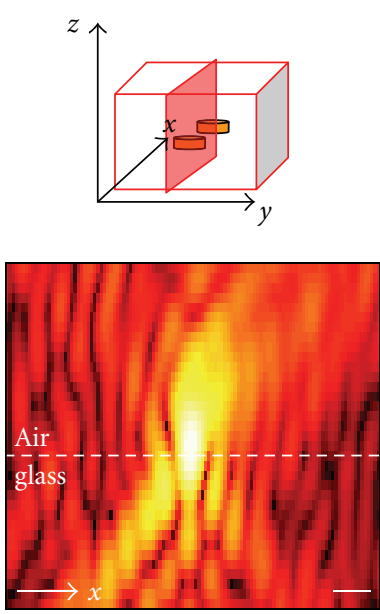

(b)

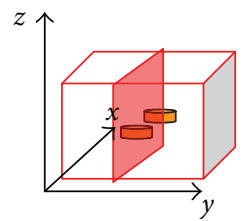

$12 \cdot 10^{5}$
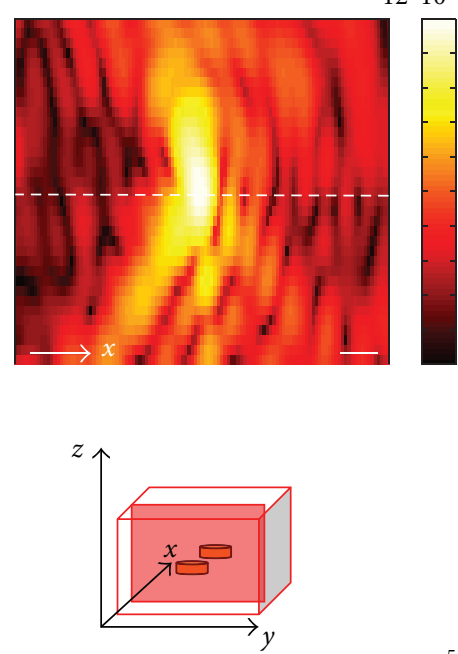

$4.5 \cdot 10^{5}$

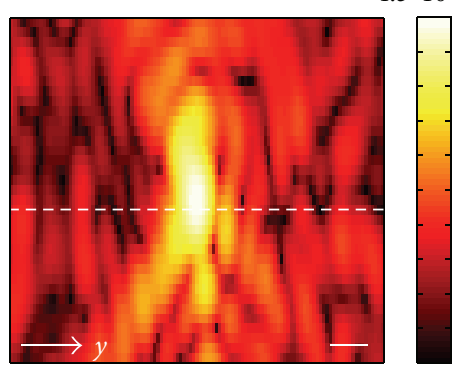

Figure 4: Intensity (arb. unit) of the scattered field reconstructed from a single hologram at $\lambda_{\text {exc }}=785 \mathrm{~nm}$ for two disks, illuminated under 2 configurations ( see schemes on the left): s-polarized excitation with the dimer axis (a) perpendicular to the plane of incidence and (b) in the plane of incidence. The color scale of the $x-y$ cross section (1st column) is linear while that of the $y-z$ and $x-z$ sections is logarithmic. The length of the scale bar is $1 \mu \mathrm{m}$.

pattern observed with the longitudinal mode. While the scattering pattern of the longitudinal mode (case (a)) extends directionally along the $z$-axis in the incident plane $(y-z$ plane), the scattering pattern of the transverse mode (case (b)) is slightly inclined towards positive $y$-values. In order to reveal finer differences between the two modes, we analyse the Fourier space of the hologram which contains information about the angular scattered intensity.

\section{Angular Scattering Pattern of Two Coupled Disks}

The emission pattern of an object can be recorded by collecting the emitted photons with an objective lens and imaging the intensity distribution in the back focal plane of the objective. These images, that display the Fourier plane, contain the angular distribution of the emitted light [29]. The intensity and the coordinates of the Fourier space are related to the angular radiation pattern as demonstrated in the literature [17]. In contrast, in digital holography, which records the amplitude and the phase of the scattered light, the Fourier plane can be obtained via Fourier transformation of the reconstructed hologram. The angular intensity distribution of the scattered light can thus be accessed in the hologram. Since the setup collects forward scattered light, the Fourier space only contains information on the angular scattering pattern in air.

Figures 5(a) and 5(b) display the Fourier planes, calculated from measurements of Figure 4, that is, the angular scattering pattern of the recorded holograms. A circular scattering pattern is observed with coordinates directly linked to the two angles $\theta$ and $\varphi$. Its radius corresponds to the maximum collection angle of $\theta_{\mathrm{NA}}=72^{\circ}$, limited by the $\mathrm{NA}=0.95$ objective.

Whereas cross-sections of the scattering pattern revealed minor differences, it is evident that the two induced modes 

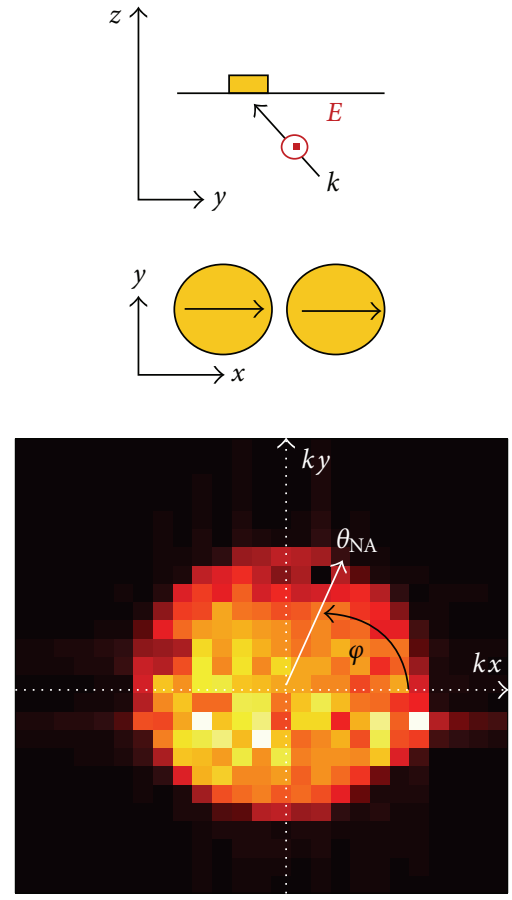

(a)
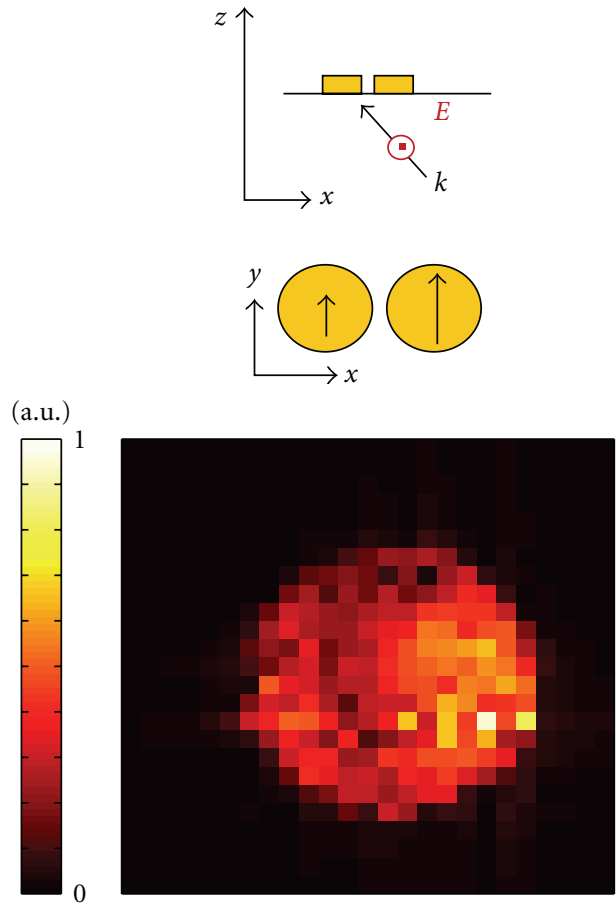

(b)

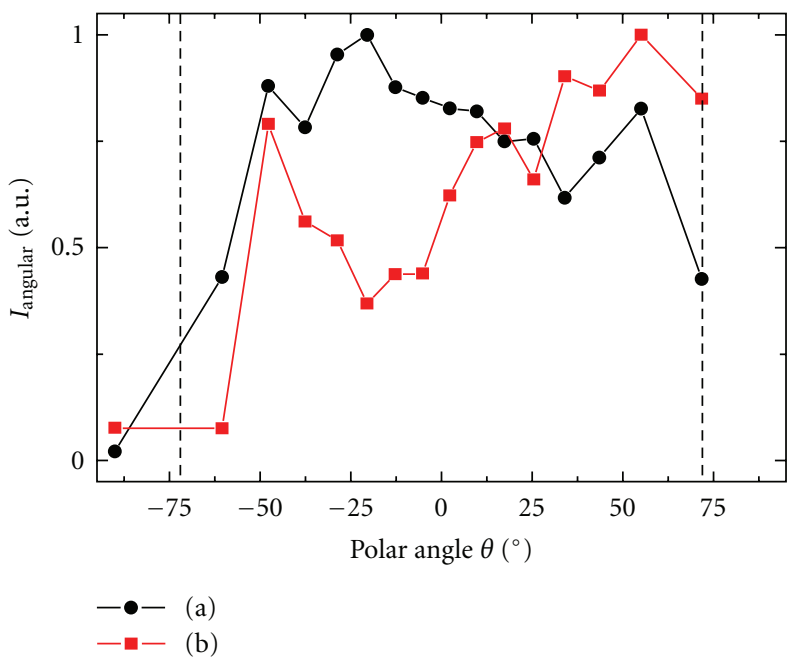

(c)

Figure 5: $(\mathrm{a}, \mathrm{b})$ Fourier planes of the recorded holograms of two coupled disks, corresponding to Figure 4 . The two axes $k_{x}$ and $k_{y}$ are indicated by dotted lines in image (a). (c) Angular normalized intensity distribution $I_{\text {angular }}$ as a function of the polar angle $\theta$ (corresponding to the $k_{x}$-axis, resp.) for the two different illumination configurations for $\varphi=0^{\circ}$, averaged over 3 lines of pixels.

differ strongly in their angular distribution. In configuration (a), the intensity is distributed homogeneously in the $k$ space, whereas in configuration (b), most of the intensity is distributed along the $+k_{x}$-axis. The graph in Figure 5(c) plots the normalized angular scattering intensity $I_{\text {angular }}$ as a function of the polar angle $\theta$, which is in fact a line plot along the $k_{x}$-axis. Due to the pixelation in the $k$-space, we obtain an angle resolution of around $8^{\circ}$. This plot confirms the images of Figures 5(a) and 5(b) with preferential light scattering in configuration (b) towards positive $\theta$ values.
We have seen that the two holograms, although very similar in the real space, reveal a profound difference in the Fourier space. This indicates different phase behaviour of the two excited modes in the nanodisk pair as expected from the near-field simulations. The symmetry of the scattering behaviour of the longitudinal mode is consistent with inphase induced dipoles. On the other hand, strong directionality in the angular scattering pattern of the transverse mode reveals the influence of phase differences between induced dipoles separated by $\lambda / 4$. 


\section{Conclusion}

We have presented a study of the spatial scattering behaviour of sub-wavelength coupled nanodisks, excited in an in-phase longitudinal or an antisymmetric transverse mode. The nature of the induced modes was estimated using near-field three-dimensional calculations which account for the total internal reflection excitation geometry used in our experimental setup. Three-dimensional far-field measurements revealed that both modes scatter light with comparable efficiencies which contradicts quasi-static approximations such as the hybridization model. The analysis of the reconstructed hologram in the Fourier plane demonstrates that the two investigated modes display strikingly different scattering patterns. In particular, the transverse mode scatters light preferentially into one half-plane, indicating that phase effects must occur in the two induced dipoles. Our study proves that the quasi-static approximation breaks down in a simple particle dimer even for center-to-center spacings smaller than $\lambda / 4$. The thorough analysis of the near-field phase effects that occur in coupled induced dipoles is necessary to fully comprehend and optimize the control offered by optical antennas on the directionality of single quantum emitters.

\section{Acknowledgments}

The authors are grateful to C. Dupuis from the LPN where the nanofabrication has been performed. They would like to acknowledge financial support from the Fondation PierreGilles de Gennes and Agence Nationale de la Recherche Grants 3D BROM (ANR 11 BS10 015 01) and TWINS (ANR 11 BS10 002 02).

\section{References}

[1] S. Nie and S. R. Emory, "Probing single molecules and single nanoparticles by surface-enhanced Raman scattering," Science, vol. 275, no. 5303, pp. 1102-1106, 1997.

[2] J. P. Camden, J. A. Dieringer, Y. Wang et al., "Probing the structure of single-molecule surface-enhanced Raman scattering hot spots," Journal of the American Chemical Society, vol. 130, no. 38, pp. 12616-12617, 2008.

[3] N. Liu, M. L. Tang, M. Hentschel, H. Giessen, and A. P. Alivisatos, "Nanoantenna-enhanced gas sensing in a single tailored focus," Nature Materials, vol. 10, pp. 631-636, 2011.

[4] P. Bharadwaj, R. Beams, and L. Novotny, "Nanoscale spectroscopy with optical antennas," Chemical Science, vol. 2, no. 1, pp. 136-140, 2011.

[5] S. S. Aćimović, M. P. Kreuzer, M. U. González, and R. Quidant, "Plasmon near-field coupling in metal dimers as a step toward single-molecule sensing," ACS Nano, vol. 3, no. 5, pp. 12311237, 2009.

[6] A. N. Grigorenko, N. W. Roberts, M. R. Dickinson, and Y. Zhang, "Nanometric optical tweezers based on nanostructured substrates," Nature Photonics, vol. 2, no. 6, pp. 365-370, 2008.

[7] W. Zhang, L. Huang, C. Santschi, and O. J. F. Martin, "Trapping and sensing $10 \mathrm{~nm}$ metal nanoparticles using plasmonic dipole antennas," Nano Letters, vol. 10, no. 3, pp. 1006-1011, 2010.
[8] P. Nordlander, C. Oubre, E. Prodan, K. Li, and M. I. Stockman, "Plasmon hybridization in nanoparticle dimers," Nano Letters, vol. 4, no. 5, pp. 899-903, 2004.

[9] W. Rechberger, A. Hohenau, A. Leitner, J. R. Krenn, B. Lamprecht, and F. R. Aussenegg, "Optical properties of two interacting gold nanoparticles," Optics Communications, vol. 220, no. 1-3, pp. 137-141, 2003.

[10] K.-H. Su, Q.-H. Wei, X. Zhang, J. J. Mock, D. R. Smith, and S. Schultz, "Interparticle coupling effects on plasmon resonances of nanogold particles," Nano Letters, vol. 3, no. 8, pp. 10871090, 2003.

[11] T. Atay, J.-H. Song, and A. V. Nurmikko, "Strongly interacting plasmon nanoparticle pairs: from dipole-dipole interaction to conductively coupled regime," Nano Letters, vol. 4, no. 9, pp. 1627-1631, 2004.

[12] P. K. Jain, W. Huang, and M. A. El-Sayed, "On the universal scaling behavior of the distance decay of plasmon coupling in metal nanoparticle pairs: a plasmon ruler equation," Nano Letters, vol. 7, no. 7, pp. 2080-2088, 2007.

[13] A. F. Koenderink, R. de Waele, J. C. Prangsma, and A. Polman, "Experimental evidence for large dynamic effects on the plasmon dispersion of subwavelength metal nanoparticle waveguides," Physical Review B, vol. 76, no. 20, Article ID 201403, 4 pages, 2007.

[14] R. de Waele, A. F. Koenderink, and A. Polman, "Tunable nanoscale localization of energy on plasmon particle arrays," Nano Letters, vol. 7, no. 7, pp. 2004-2008, 2007.

[15] J. N. Farahani, D. W. Pohl, H.-J. Eisler, and B. Hecht, "Single quantum dot coupled to a scanning optical antenna: a tunable superemitter," Physical Review Letters, vol. 95, no. 1, pp. 1-4, 2005.

[16] T. Kosako, Y. Kadoya, and H. F. Hofmann, "Directional control of light by a nano-optical Yagi-Uda antenna," Nature Photonics, vol. 4, no. 5, pp. 312-315, 2010.

[17] A. G. Curto, G. Volpe, T. H. Taminiau, M. P. Kreuzer, R. Quidant, and N. F. van Hulst, "Unidirectional emission of a quantum dot coupled to a nanoantenna," Science, vol. 329, no. 5994, pp. 930-933, 2010.

[18] N. Bonod, A. Devilez, B. Rolly, S. Bidault, and B. Stout, "Ultracompact and unidirectional metallic antennas," Physical Review B, vol. 82, no. 11, Article ID 115429, 6 pages, 2010.

[19] B. Rolly, B. Stout, S. Bidault, and N. Bonod, "Crucial role of the emitter-particle distance on the directivity of optical antennas," Optics Letters, vol. 36, no. 17, pp. 3368-3370, 2011.

[20] J. Dorfmüller, D. Dregely, M. Esslinger et al., "Near-field dynamics of optical Yagi-Uda nanoantennas," Nano Letters, vol. 11, no. 7, pp. 2819-2824, 2011.

[21] P. Olk, J. Renger, M. T. Wenzel, and L. M. Eng, "Distance dependent spectral tuning of two coupled metal nanoparticles," Nano Letters, vol. 8, no. 4, pp. 1174-1178, 2008.

[22] S.-C. Yang, H. Kobori, C.-L. He et al., "Plasmon hybridization in individual gold nanocrystal dimers: direct observation of bright and dark modes," Nano Letters, vol. 10, no. 2, pp. 632637, 2010.

[23] B. Rolly, B. Stout, and N. Bonod, "Metallic dimers: when bonding transverse modes shine light," Physical Review B, vol. 84, no. 12, article 125420, 8 pages, 2011.

[24] T. Shegai, S. Chen, V. D. Miljković, G. Zengin, P. Johansson, and M. Käll, "A bimetallic nanoantenna for directional colour routing," Nature Communications, vol. 2, no. 1, article 481, 2011.

[25] M. Atlan, M. Gross, P. Desbiolles, E. Absil, G. Tessier, and M. Coppey-Moisan, "Heterodyne holographic microscopy of gold particles," Optics Letters, vol. 33, no. 5, pp. 500-502, 2008. 
[26] S. Y. Suck, S. Collin, N. Bardou, Y. de Wilde, and G. Tessier, "Imaging the three-dimensional scattering pattern of plasmonic nanodisk chains by digital heterodyne holography," Optics Letters, vol. 36, no. 6, pp. 849-851, 2011.

[27] E. Cuche, P. Marquet, and C. Depeursinge, "Spatial filtering for zero-order and twin-image elimination in digital off-axis holography," Applied Optics, vol. 39, no. 23, pp. 4070-4075, 2000.

[28] E. Absil, G. Tessier, M. Gross et al., "Photothermal heterodyne holography of gold nanoparticles," Optics Express, vol. 18, no. 2, pp. 780-786, 2010.

[29] M. A. Lieb, J. M. Zavislan, and L. Novotny, "Single-molecule orientations determined by direct emission pattern imaging," Journal of the Optical Society of America B, vol. 21, no. 6, pp. 1210-1215, 2004. 

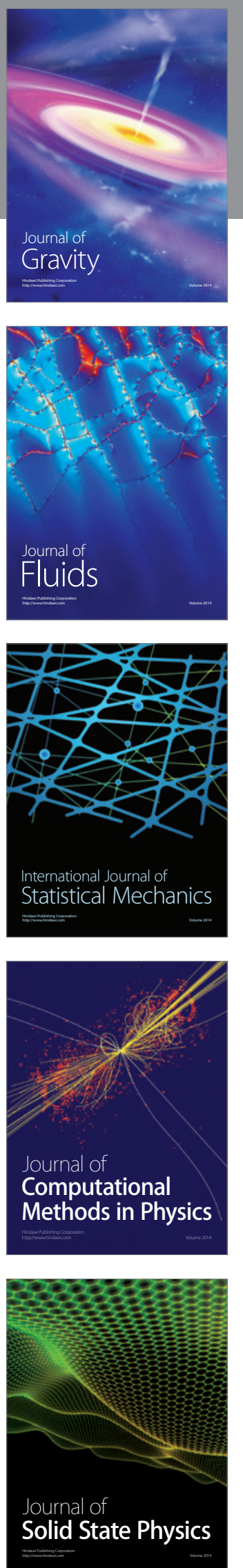

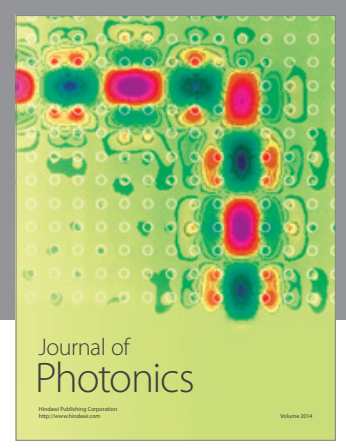

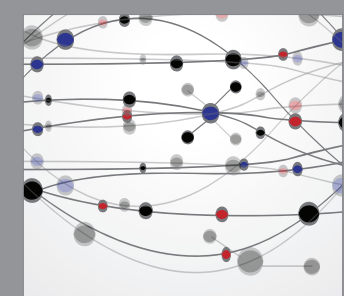

The Scientific World Journal
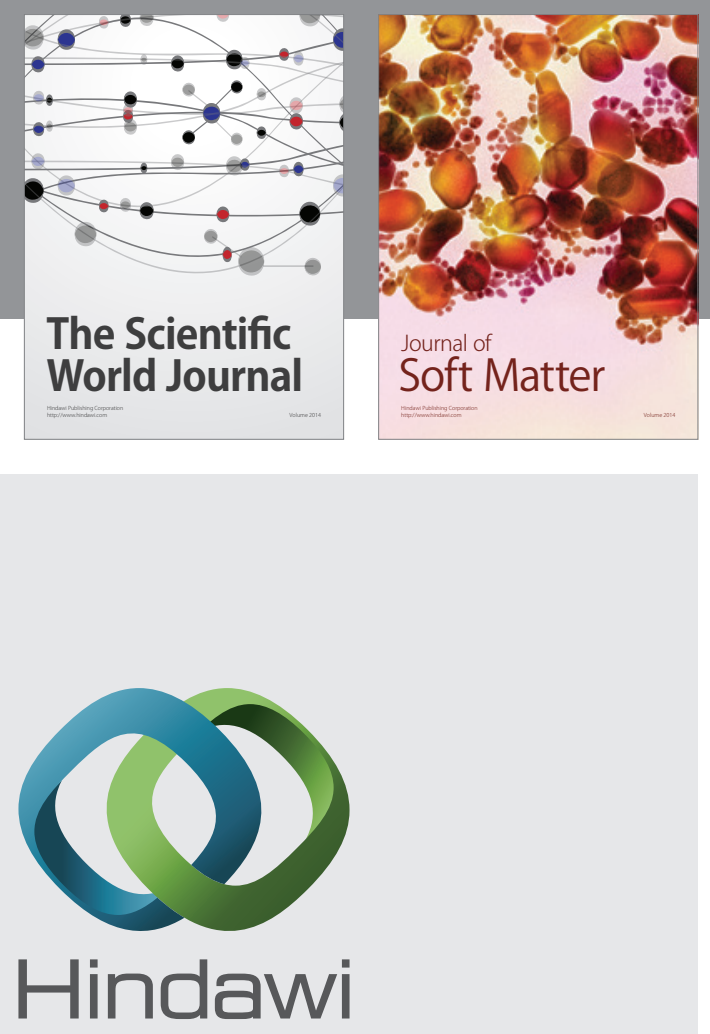

Submit your manuscripts at

http://www.hindawi.com
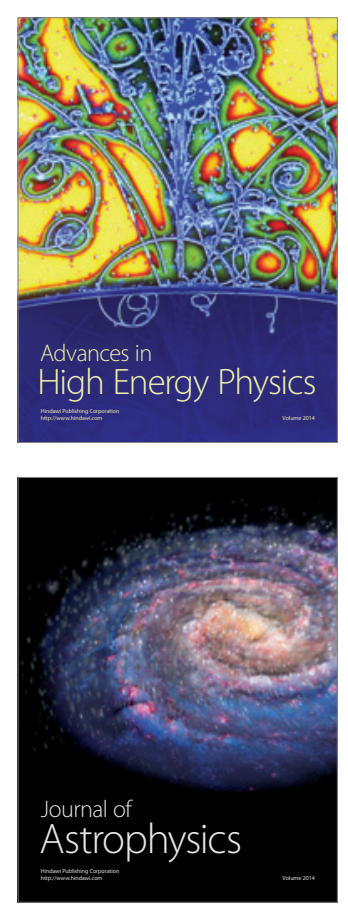
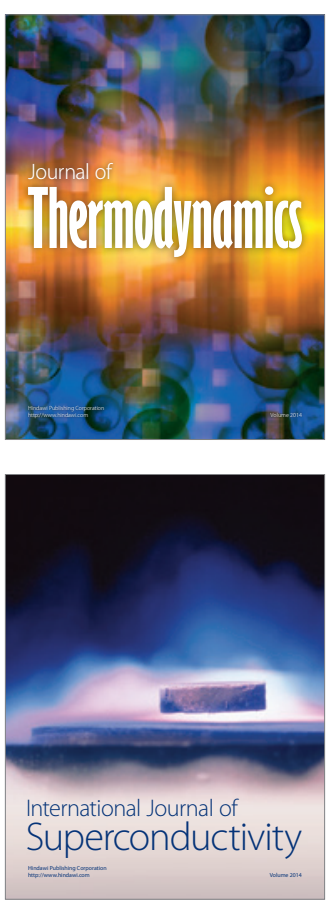
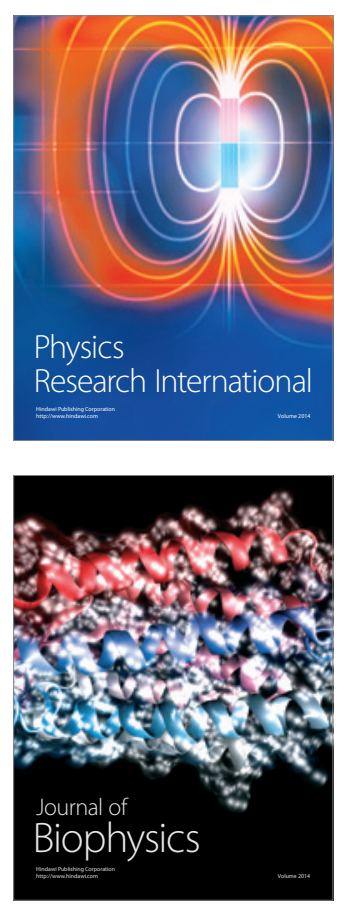
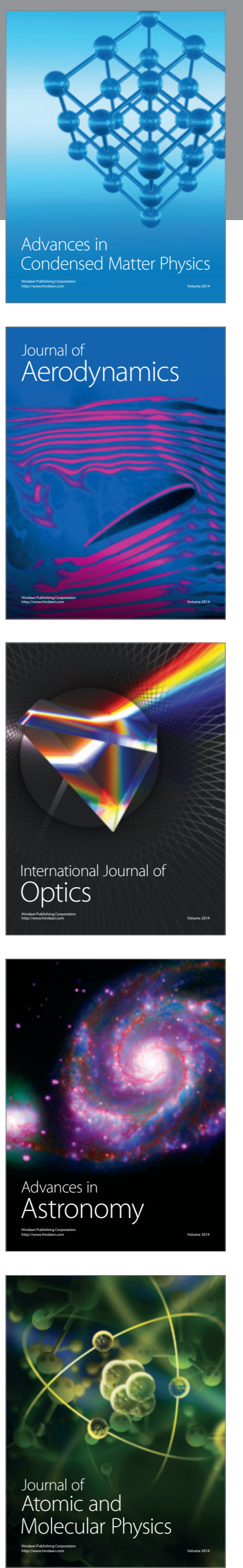